\title{
The Application of Indicators to Assess the Severity of Seasonal Influenza Epidemics in Democratic Republic of Congo, 2015 to 2019
}

\author{
Saleh Muhemedi1 ${ }^{*}$, Paul Lusamba ${ }^{1}$, Jean-Claude Changachanga ${ }^{2}$, Leopold Lubula ${ }^{3}$, \\ Edith Nkwembe ${ }^{2}$, Pelagie Babakazo ${ }^{1}$
}

${ }^{1}$ Kinshasa School of Public Health, Kinshasa University, Kinshasa, DRC

${ }^{2}$ National Institute of Biomedical Researches, Ministry of Health, Kinshasa, DRC

${ }^{3}$ Department of Surveillance Disease, Ministry of Health, Kinshasa, DRC

Email: *muhemedi2004@yahoo.fr

How to cite this paper: Muhemedi, S., Lusamba, P., Changachanga, J.-C., Lubula, L., Nkwembe, E. and Babakazo, P. (2022) The Application of Indicators to Assess the Severity of Seasonal Influenza Epidemics in Democratic Republic of Congo, 2015 to 2019. Open Journal of Respiratory Diseases, 12 , $1-14$.

\section{https://doi.org/10.4236/ojrd.2022.121001}

Received: January 28, 2022

Accepted: February 22, 2022

Published: February 25, 2022

Copyright $\odot 2022$ by author(s) and Scientific Research Publishing Inc. This work is licensed under the Creative Commons Attribution International License (CC BY 4.0).

http://creativecommons.org/licenses/by/4.0/

\section{(c) (i) Open Access}

\begin{abstract}
Introduction: Among the lessons learned from the 2009 influenza pandemic was the lack of a robust, standardized method that would allow a timely assessment of the severity of pandemic influenza. To remedy this deficiency, WHO has set up an evaluation tool based on the following indicators: 1) transmissibility, 2) seriousness of disease, and 3) impact of the influenza pandemic. By using this pandemic influenza severity assessment (PISA) tool, this study aimed to evaluate the severity of DRC influenza seasons between 2015 and 2019 to better prepare the country against the possible occurrence of an influenza pandemic. Methods: We performed a secondary data analysis from the DRC Influenza routine surveillance. We only explored the transmissibility among PISA indicators. Results: The results of our study showed that the DRC influenza seasons had two waves. The first went from the 40th week to the 10th week, with a peak at the 50th week, and the second wave ran from the 15th week to the 40th, with a peak at the 19th week. There was an inter-wave period between the 10th and 15th weeks. Of all the studied seasons, $42.8 \%$ were characterized by low intensity, $33.3 \%$ by moderate intensity, $19.0 \%$ by high intensity, and $4.8 \%$ by extraordinary intensity. Conclusion: The use of the PISA transmissibility indicator has contributed to better understanding influenza seasons in the Democratic Republic of Congo.
\end{abstract}

\section{Keywords \\ PISA, Influenza, DRC}

\section{Introduction}

Influenza is an acute respiratory infection that occurs worldwide. It is a real pub- 
lic health problem, affecting $5 \%-10 \%$ of adults and $20 \%-30 \%$ of children each year, and leading to around five million cases of serious illness and 290,000 650,000 cases of death [1].

The disease regularly appears in countries with temperate climates as seasonal epidemics in late autumn and during winter, causing a significant human and economic burden [2] [3] [4] [5] [6] [7]. In warm countries with equatorial, tropical, or subtropical climates, cases of influenza appear throughout the year, with more or less irregular outbreaks. Little is known about the existence of a seasonal pattern of influenza transmission over the year. However, with the development of methods for calculating epidemic thresholds, some countries in these regions have managed to determine the beginning, the duration, and the end of their influenza seasons [8] [9] [10]. Furthermore, the burden of influenza is not well known, although there is growing evidence of similarity between temperate regions [11] [12] [13] [14]. Despite temporary variations in influenza outbreaks, the existence of seasonality has been established for some countries.

What is generally known is that climatic, environmental, social behavioral, and virological factors contribute to the occurrence of influenza epidemics whose severity and duration are subject to annual variations with no foreseeable cause [15]-[21].

One of the lessons learned from the 2009 influenza pandemic was the lack of a robust and standardized method to assess the severity of pandemic influenza in a timely manner. To remedy this deficiency, the World Health Assembly held in 2011 recommended to WHO the setting up of a tool that should permit the assessment both the severity of each seasonal influenza epidemic, or pandemic and to determine its chronology, scale, and importance as well as control measures to be urgently implemented. To solve this issue, WHO has developed a pandemic influenza severity assessment (PISA) tool using three indicators: 1) transmissibility, 2) seriousness of disease, and 3) impact [22] [23]. The tool thus created, should set staggered thresholds based on historical epidemiological and virological data.

Apart from a pandemic, the PISA tool should also able to address seasonal influenza by determining its beginning, duration, end, intensity, number of waves and their peaks, in order to facilitate decision-making by the health authorities [24]. This tool recommends that transmissibility and impact indicators be measured from weekly rates while the seriousness indicator be measured from cumulative values.

Although the PISA tool has defined indicators to measure the severity of seasonal or pandemic influenza, some methods are used for applying those indicators according to the nature of dataset availability. The most well-known are the Moving Epidemic Method [25] [26] [27] and the WHO Average Curves Method. In addition to these two methods, the country-specific statically empirical method (CSED) is also used [28].

Thanks to the PISA tool, many countries are preparing to face a possible influenza pandemic. That's why we have conducted this study as a preparedness 
exercise for the DRC in order to assess influenza seasons between 2015 and 2019. This assessment aimed to determine the beginning, duration, and ending of influenza seasons as well as the number of waves per season and the intensity of thresholds.

\section{Methods}

\subsection{Influenza Surveillance in the DRC}

The Democratic Republic of Congo is a vast country in Central Africa of $2,345,000 \mathrm{~km}^{2}$ with $88,567,000$ inhabitants. It is a warm country with two main climates: an equatorial climate characterized by rains throughout the year and a tropical climate with a 9-month long rainy season and a 3-month short dry season. In 2007, a sentinel influenza surveillance system was implemented, then gradually expanded to reach 12 sentinel sites in 2016 across the country. Bucco-pharyngeal and nasopharyngeal samples are taken from ILI and SARI and sent to the national influenza laboratory for testing. In addition to sentinel influenza surveillance, the country has a routine surveillance that deals with the notification of around 30 illnesses. The aim of influenza surveillance is both to detect the viral types and subtypes of influenza circulating in the country and to determine the burden of this disease to enable health authorities to take adequate control measures.

\subsection{Data Source}

This study used epidemiological and virological data from sentinel surveillance and routine surveillance for the period from the 1st week of 2015 to the 39th week of 2019. A total of four types of datasets were used: 1) number of new outpatients, 2) number of ILI patients, 3) number of samples collected from ILI patients for laboratory testing, and 4) number of influenza positive ILI samples among tested samples. All the tests were carried out at the national influenza laboratory using the PCR technique.

During the period considered in our study, a total of 9445 ILI cases out of a total of 254,586 outpatients were recorded. Among the ILI cases, 3958 were tested in the laboratory, of which 317 were positive for the influenza virus. By definition, an ILI case was considered to be any patient admitted to an outpatient clinic with a fever of $38^{\circ} \mathrm{C}$ or higher, or with a history of fever, cough, or sore throat within 10 days. The DRC surveillance system generated these data, which are available at https://worldhealthorg.shinyapps.io/averagecurves/.

\subsection{Analysis Process}

To assess the severity of the influenza seasons from 2015 to 2019, we have chosen the indicator of transmissibility relating to ILI cases. The evaluation process followed four steps described by WHO, notably: 1) choice of parameters to be used; 2) definition of the thresholds for each parameter selected; 3) application of the thresholds to assess the transmissibility of influenza; and 4) communica- 
tion of assessment results [22].

\section{1) Choice of parameters}

For the first step, we chose three parameters: the syndromic ILI parameter, the virological ILI parameter, and the composite ILI parameter. These parameters were calculated weekly from the formulas shown in Table 1.

2) Establishment of seasonal thresholds and determination of the intensity of transmission or impact

For these steps, we used the WHO average curves method, which is posted on the web as an application at https://worldhealthorg.shinyapps.io/averagecurves/.

This application makes it possible to describe influenza activities for each year separately or by shifted year. Our preference was to use shifted years given the characteristics of our flu season, which spans two years. Where the tool did not allow the use of staggered years, we used separated years. This tool is also able to establish a comparison within the same graph between the average curve of influenza activity for previous years (2014/2015, 2015/2016, 2016/2017, 2017/2018), called the "average epidemic curve", and the epidemic curve of the year under surveillance (2018/2019), based on 4 thresholds: the epidemic threshold, the moderate threshold, the high threshold, and the extraordinary threshold. In addition, this application has the ability to determine the value of epidemic peaks and wave intensity.

The epidemic threshold was obtained by calculating the historical median rate per week. Other thresholds of the average curve were calculated on the basis of the historical peaks and the mean and standard deviations of their intensities. Using the normal distribution, the 40,90 , and 97.5 percent intervals were used to calculate the moderate, high, and extraordinary thresholds, respectively. Intensity thresholds were calculated separately for each wave. The operation of this application has been adapted from the WHO Manual of Global Influenza Epidemiological Surveillance Standards [24].

\subsection{Ethical Considerations}

Data used in this paper comes from the WHO FluNet and FluID networks for

Table 1. Parameters of the transmissibility indicators and their calculation methods.

\begin{tabular}{|c|c|c|}
\hline Indicator & Parameters & Calculation \\
\hline \multirow{3}{*}{ Transmissibility } & syndromic ILI & $\begin{array}{l}\text { Number of notified ILI } \\
\text { cases }^{\star} 100 / \text { Total of outpatients (a) } \\
\text { (a) }=\text { Proportion of notified ILI cases }\end{array}$ \\
\hline & virological ILI & $\begin{array}{l}\text { Number of positive ILI cases }{ }^{\star} 100 / \text { Total } \\
\text { of tested ILI cases (b) } \\
\text { (b) = Positivity rate of ILI cases }\end{array}$ \\
\hline & $\begin{array}{l}\text { Composite ILI or } \\
\text { product of syndromic } \\
\text { and virological ILI }\end{array}$ & $\begin{array}{l}\text { Proportion of notified ILI } \\
\text { cases }^{\star} \text { Positivity rate of ILI cases }\left\{(a)^{\star}(b)\right\}\end{array}$ \\
\hline
\end{tabular}


influenza surveillance. They were generated by the DRC Ministry of Health through the office of Surveillance Diseases. We required and obtained approval from the Kinshasa University IRB committee to use them.

\section{Results}

\subsection{Transmissibility}

\subsubsection{Graphical Representation of the Data}

In relation to transmissibility, Figure 1 includes three curves representing syndromic, virological, and composite ILI parameters from 2015 to 2018/2019. Data are expressed per cent, $1 / 100$ for curve $1 \mathrm{a}, 1 / 100$ for curve $1 \mathrm{~b}$, and $1 / 10,000$ for curve $1 \mathrm{c}$, per week.

The examination of the lagraph shows a sawtooth trend with a high peak of cases recorded in 2017. On the other hand, the $1 \mathrm{~b}$ curve shows several peaks, of which the highest are located in 2015 and 2018. This curve shows an interruption in the 2017 season. The $1 \mathrm{c}$ curve follows almost the same trend as curve $1 \mathrm{~b}$, but with fewer peaks. Those two curves show the limitation of flu seasons.

\subsubsection{Establishment of Seasonal Thresholds and Determination of Transmission Intensity or Impact}

For the three ILI parameters, epidemiological curves of the 2014/2015, 2015/2016, 2016/2017, 2017/2018, and 2018/2019 influenza seasons were produced based on the epidemiological weeks as shown in Figure 2. Sub Figures 2(a)-(c) represent, respectively, syndromic, virological, and composite data. All these curves were aligned to the median value of their respective peaks as described in the methodology. Thus, the alignment was carried out at week 46 for

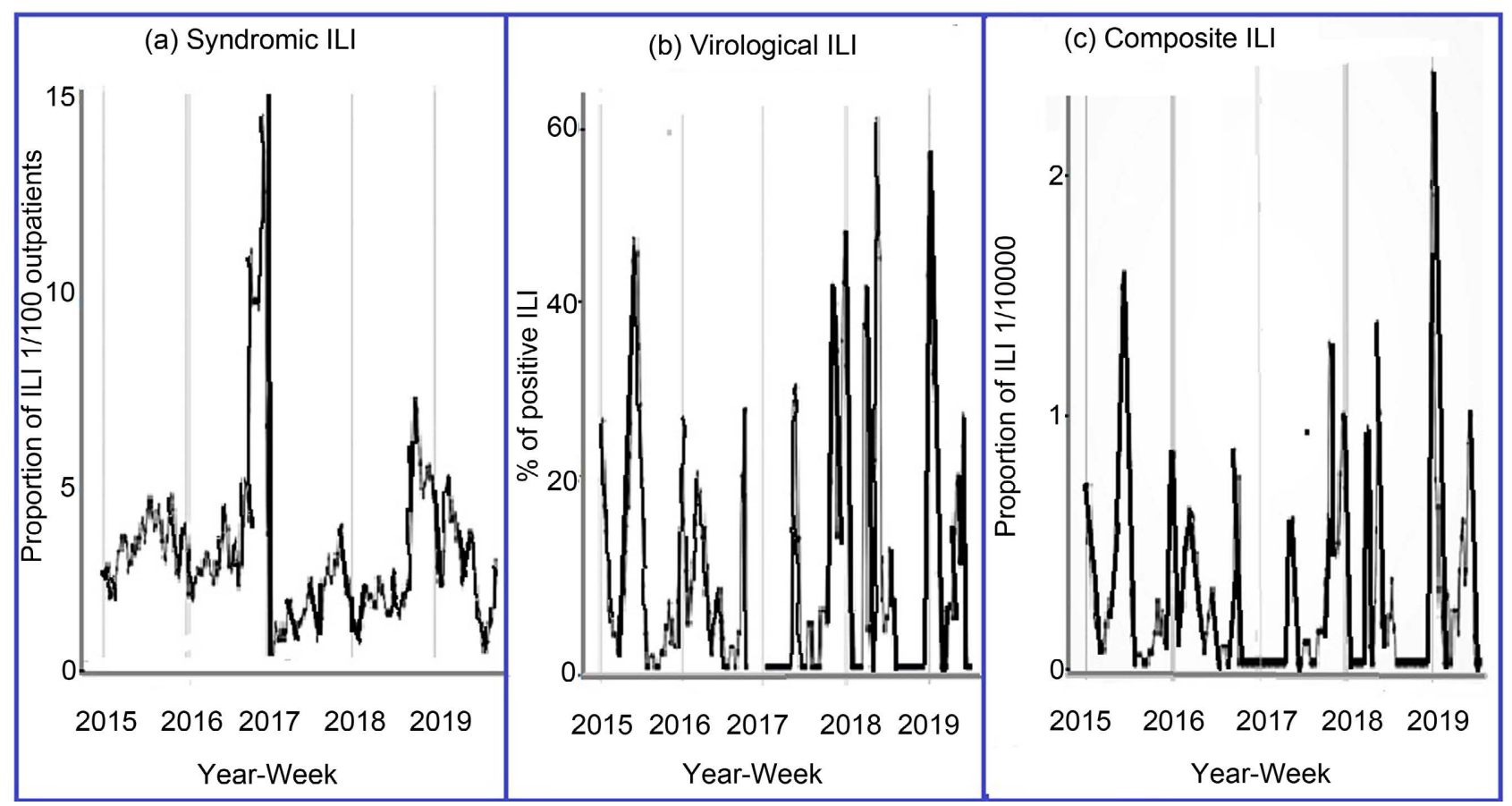

Figure 1. Time series of ILI. 


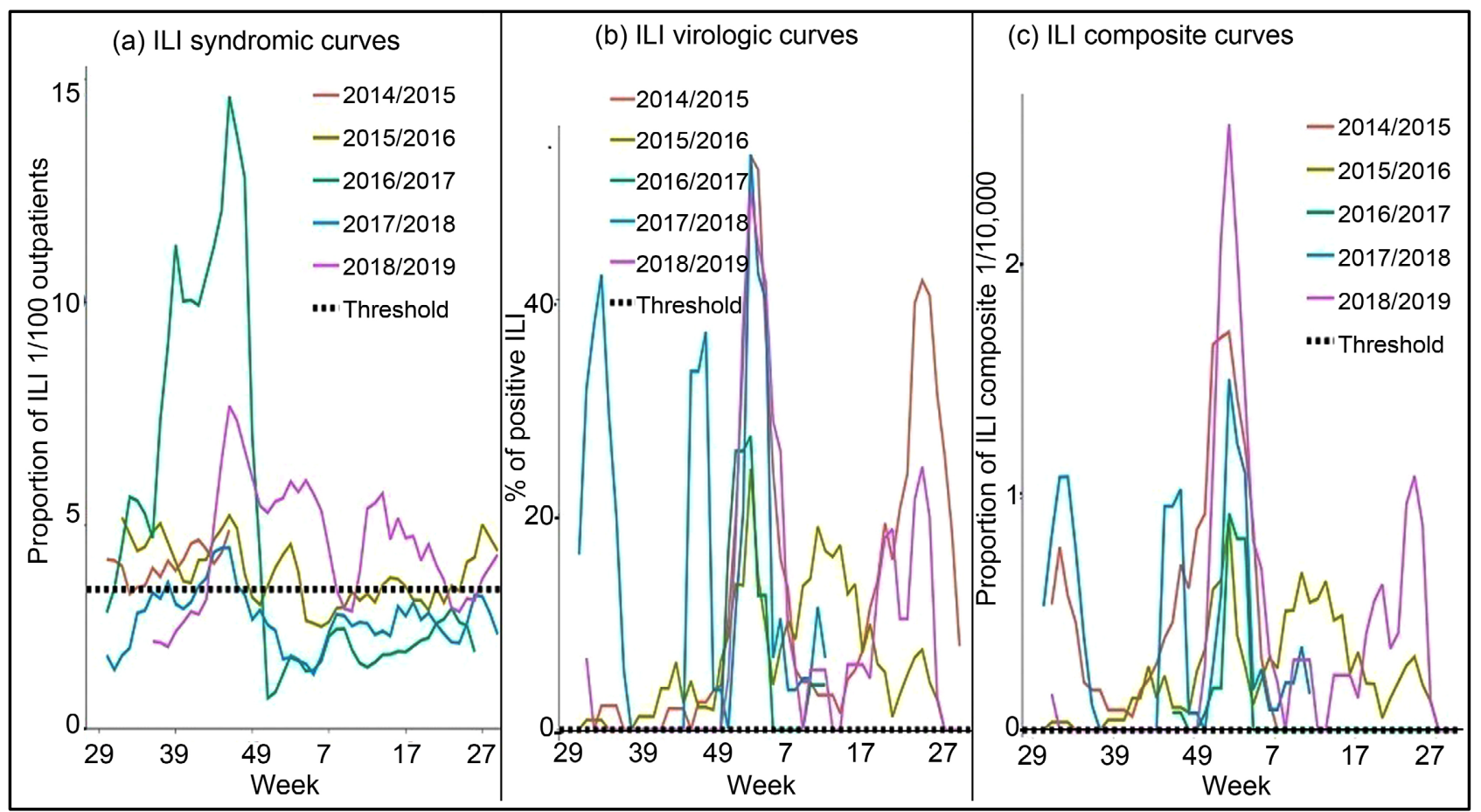

Figure 2. Seasonal aligned curves.

curve $2 \mathrm{a}$, and at week 52 for curves $2 \mathrm{~b}$ and $2 \mathrm{c}$. This figure also shows that the epidemic threshold is located at 3.38 for the syndromic data, reflecting pre- and post-epidemic periods. For the virological and composite parameters, the epidemic threshold is equal to 0 . The details of the influenza activity during the different seasons are shown in Table 2.

This table shows the existence of two waves for each influenza season. Each wave has a peak with a certain value and is located during a specific week. The wave also has an intensity that reflects the influenza activity.

For a total of four seasons, eight waves were identified. However, some data is missing. These are the data for the syndromic and composite ILI parameters from wave 1 of the 2014/2015 season, as well as the virological ILI parameter from wave 1 of the 2016/2017 season.

The wave intensity assessment highlighted 21 ratings for all 3 parameters. "L" denotes low activity level and represents 9 waves out of $21 \%$ or $42.8 \%$, moderate activity level " $\mathrm{M}$ " had a total of 7 waves out of $21 \%$ or $33.3 \%$, high activity level "H" scored 4 waves out of $21 \%$ or $19.0 \%$, and finally, extraordinary activity level "E" one wave out of $21 \%$ or $4.8 \%$.

The average of the activities of the epidemiological curves of the previous seasons (ranging from the 2014/2015 season to the 2017/2018 season) is represented by an average epidemiological curve for each of the three parameters studied as shown in Figure 3; Figure 3(a) for the syndromic ILI parameter, Figure 3(b) for the virological ILI parameter, and Figure 3(c) for the composite ILI parameter. For each parameter, the epidemiological curve of the influenza season under surveillance $(2018 / 2019)$ is compared to the average epidemiological 
Table 2. Characteristics of waves according to Influenza seasons.

\begin{tabular}{|c|c|c|c|c|c|c|c|c|c|}
\hline ILI parameter & Wave characteristics & \multicolumn{2}{|c|}{$2014 / 2015$} & \multicolumn{2}{|c|}{$2015 / 2016$} & \multicolumn{2}{|c|}{$2016 / 2017$} & \multicolumn{2}{|c|}{$2017 / 2018$} \\
\hline \multirow{4}{*}{$\begin{array}{l}\text { Syndromic } \\
\text { Parameter }\end{array}$} & Wave number & & 2 & 1 & 2 & 1 & 2 & 1 & 2 \\
\hline & Peak week & & 30 & 44 & 36 & 49 & 36 & 46 & 26 \\
\hline & Peak value & & 5.59 & 5.66 & 6.18 & 18.7 & 4.01 & 5.89 & 4.28 \\
\hline & Wave intensity & & M & $\mathrm{L}$ & $\mathrm{H}$ & $\mathrm{H}$ & $\mathrm{L}$ & $\mathrm{L}$ & M \\
\hline \multirow{4}{*}{$\begin{array}{l}\text { Virological } \\
\text { parameter }\end{array}$} & Wave number & 1 & 2 & 1 & 2 & & 2 & 1 & 2 \\
\hline & Peak week & 51 & 22 & 9 & 14 & & 19 & 51 & 19 \\
\hline & Peak value & 75.00 & 58.82 & 28.57 & 12.50 & & 44.44 & 63.64 & 100.00 \\
\hline & Wave intensity & $\mathrm{L}$ & $\mathrm{H}$ & $\mathrm{L}$ & $\mathrm{L}$ & & M & $\mathrm{H}$ & $\mathrm{E}$ \\
\hline \multirow{4}{*}{$\begin{array}{l}\text { Composite } \\
\text { parameter }\end{array}$} & Wave number & & 2 & 1 & 2 & 1 & 2 & 1 & 2 \\
\hline & Peak week & & 23 & 9 & 14 & 44 & 19 & 44 & 19 \\
\hline & Peak value & & 1.7 & 0.68 & 0.49 & 0.00 & 0.63 & 1.38 & 1.58 \\
\hline & Wave intensity & & M & M & $\mathrm{L}$ & $\mathrm{L}$ & $\mathrm{L}$ & M & M \\
\hline
\end{tabular}

L: low; M: Moderate; H: High; E: Extraordinary.

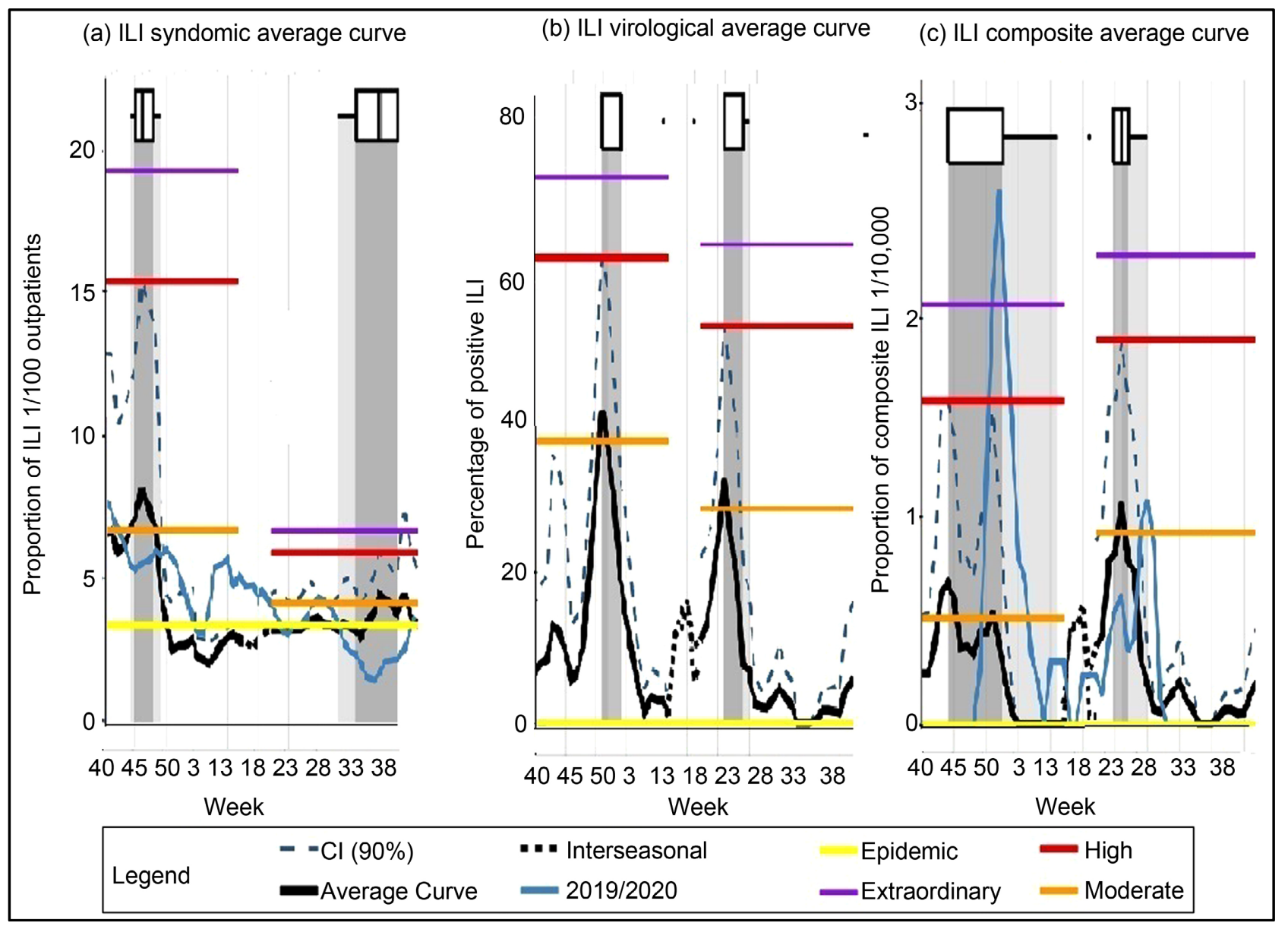

Figure 3. ILI average curves compared to 2018/2019 seasonal epidemic curves. 
curve, reflecting all the influenza activities of previous seasons.

At the level of each sub-graph, the average epidemic curve is represented in black and the season under surveillance in blue. The average epidemic curve shows a typical influenza season, in terms of weeks and intensity. The distribution of past seasonal peaks is represented by a whisker box and vertical shading. Thresholds representing the intensity of activity are indicated by colored cross lines.

The mean curve was calculated over two periods: from week 40 to week 15, and from week 10 to week 40. Between the two periods, there is a 5-week overlap period, from week 10 to week 15 , which is indicated on the epidemiological curve by black dotted lines. The severity of the season was assessed from the maximum intensity of each wave, as shown in Table 2.

According to the syndromic ILI parameter shown in subgraph 3a, the epidemic period is from the 40th week to the 50th week, when the mean epidemic curve crosses the epidemic threshold. The peak for this period is moderate and occurs in week 46 . From the 50th week, a post epidemic period occurs which continues until the 30th week, then a short epidemic period of low intensity appears which continues until the 39th week.

With the virological ILI parameter, the first wave begins at week 40 with a moderate intensity peak at week 50 , then decreases in intensity until the inter-wave period extends from week 10 to 15 . The second wave begins from week 15 to week 39, with a moderate peak in intensity at week 19 .

According to the composite parameter, the season starts at week 40 and peaks at week 45 , then starts again at week 13 with a peak at week 19, and ends at week 39. An inter-wave period was observed between the 10th and 15th week.

For the 2018/2019 season under surveillance, the trend of the syndromic ILI curve is almost similar to that of the average epidemic curve, except for the post epidemic period, which is quite short. This similarity is also remarkable for the virological ILI parameter. In contrast, the 2018-2019 composite epidemic curve is singular because it reached an extraordinary threshold in the first wave and a high threshold in the second wave.

As in the influenza seasons, four thresholds were calculated for the average epidemic curve, as shown in Table 3. In total, four thresholds were calculated

Table 3. Threshold of the average epidemic curve by parameter and by wave from $2014 / 2015$ to $2018 / 2019$ influenza season.

\begin{tabular}{ccccccc}
\hline \multirow{2}{*}{ Threshold } & \multicolumn{2}{c}{$\begin{array}{c}\text { Syndromic } \\
\text { parameter }\end{array}$} & \multicolumn{2}{c}{$\begin{array}{c}\text { Virological } \\
\text { parameter }\end{array}$} & \multicolumn{2}{c}{$\begin{array}{c}\text { Composite } \\
\text { parameter }\end{array}$} \\
\cline { 2 - 7 } & Wave 1 & Wave 2 & Wave 1 & Wave 2 & Wave 1 & Wave 2 \\
\hline Epidemic & 3.38 & 3.38 & 0.00 & 0.00 & 0.00 & 0.00 \\
Moderate & 6.68 & 4.14 & 33.7 & 29.6 & 0.51 & 0.93 \\
High & 15.40 & 5.80 & 60.8 & 56.3 & 1.58 & 1.88 \\
Extraordinary & 19.20 & 6.66 & 72.7 & 68.1 & 2.05 & 2.30 \\
\hline
\end{tabular}


and established as shown in Table 3 according to the three parameters. For the three average curves, the maximum threshold was moderate for all three parameters. The epidemic thresholds for the first wave are identical to those of the second for the three parameters studied. On the other hand, the thresholds are higher for the first wave compared to the second for the syndromic ILI and virological ILI parameters, while they are lower for the composite ILI parameter.

\section{Discussion}

In order to determine the influenza season in DRC, we used the WHO method with the WHO average curve web application. This tool allowed us to evaluate one of the three PISA indicators, namely the transmissibility of influenza infection. The purpose of this tool was to produce an average epidemiological curve from historical data of previous years to be compared with the epidemiological curve of the year under surveillance. To do this, we used data from the influenza seasons from 2014/2015 to 2017/2018 that we compared with the 2018/2019 influenza season. In addition to producing the epidemiological curve, the tool allowed us to determine different thresholds as well as the intensity of different influenza seasons. To do so, we used three parameters: syndromic ILI, virological ILI, and composite ILI. A total of four thresholds were established to mark the beginning of the season. These are the epidemic, moderate, high, and extraordinary thresholds. From these thresholds, levels of influenza activity have been established. These are the low, moderate, high, and extraordinary levels.

There are other methods to evaluate the severity of influenza seasons, such as the Moving Epidemic Method (MEM), but we chose the WHO method because of its simplicity, its ability to manipulate virological data [29], and, above all, the use of the algorithm allowing the division of the influenza season into two periods.

Subfigure 1a of our results on the syndromic ILI parameter showed that the influenza seasons were not clearly identifiable, unlike the virological and composite ILI parameters. These results are in contrast with those of studies carried out in Europe [30] and the United States [31] which showed that the syndromic ILI parameter was able to monitor seasonal influenza activity. However, they are similar to those found in a study in Cambodia [32]. This similarity is further reinforced by the fact that the Cambodia study, like ours, used the WHO method and that both Cambodia and the DRC are in the tropics.

The curve $1 \mathrm{~b}$ of the virological ILI time series showed a break at its base during the 2016/2017 influenza season. This break is due to the interruption of sample collection and analysis at the National Influenza Laboratory between October 2016 and January 2017, occasioned by the shortage of lab reagents and supplies. This is illustrated by the absence of virological data in the first wave of the 2016/2017 season in Table 2.

Average epidemic curves showed two waves from the $40^{\text {th }}$ to $39^{\text {th }}$ week of the next year, with an inter-wave period between the $10^{\text {th }}$ and the $15^{\text {th }}$ week. The influenza outbreaks correspond to the rainy season, which spans from September 
to June each year [33]. The first wave that runs from week 40 to week 10 corresponds in Europe to the winter epidemic period, which extends from the $52^{\text {th }}$ week to the we $8^{\text {th }}$ week, based on data from 13 influenza seasons from 2001 to 2014 in 30 countries [30].

For good reason, determining the onset of an influenza epidemic and its intensity is of great importance. It allows the health system to prepare by developing a contingency plan and allocating necessary resources such as vaccines, antivirals, and communication tools [34]. It also allows for intensified epidemiological surveillance and monitoring of high-risk individuals [35]. Setting the epidemic threshold is very important in determining the beginning and end of an epidemic. The other thresholds mentioned above are used to monitor the intensity of the epidemic and to alert the surveillance system [35].

The DRC started posting these data on the FluNet system in 2010. These are virological data from the analysis of samples of suspected influenza cases from sentinel surveillance sites. These samples were collected using the ILI and SARI case definitions adapted to those of the WHO. During the 2014-2015 season, syndromic data were collected and posted to the FluID system on a weekly basis. In this study, we aimed to compare syndromic data with virological data, but we excluded virological data prior to 2014. In addition, we were limited to assessing only one of the three PISA indicators, which is transmissibility, using the ILI data source. Furthermore, this study is the first attempt to assess the transmissibility of influenza in the DRC. We would have liked to compare our results to those of Central African countries, but they are not currently available. Despite its limitations, we are convinced that our results are valid for informing the influenza surveillance system in the DRC.

\section{Limits}

The DRC started posting these data in the FluNet system in 2010. These are virological data from the analysis of samples of suspected influenza cases from sentinel surveillance sites. These samples were collected using the ILI and SARI case definitions adapted to those of the WHO. It was from the 2014/2015 season that syndromic data were collected and posted weekly to the FluID system. As for this study, we sought to compare syndromic data with virological data. We did not include virological data from before 2014. In addition, we were limited to assessing only one of the three PISA indicators which is transmissibility using the ILI data source. Furthermore, this study is a first attempt to assess the transmissibility of influenza in DRC. We would have liked to compare our results with those of countries in the Central African region, which are not currently available. Despite its limitations, we are convinced that our results are valid for informing the influenza surveillance system in DRC.

\section{Acknowledgements}

We thank the sentinel surveillance sites in hospitals and health centers, the Na- 
tional Influenza Laboratory, the Directorate of Epidemiological Surveillance, the Kinshasa School of Public Health and the CDC/DRC for their contribution to the influenza surveillance system in the DRC.

\section{Funding}

None.

\section{Conflicts of Interest}

No conflicts of interest were declared. None of the authors of this article is affiliated with any organization or lobby that has any actual or potential interest in this study.

\section{References}

[1] World Health Organization (2016) Influenza Seasonal Factsheet N211. https://www.who.int/health-topics/influenza-seasonal\#tab=tab 1

[2] Lafond, K.E., Nair, H., Rasooly, M.H., et al. (2016) Global Role and Burden of Influenza in Pediatric Respiratory Hospitalizations, 1982-2012: A Systematic Analysis. PLoS Medicine, 13, e1001977.

https://doi.org/10.1371/journal.pmed.1002060 https://journals.plos.org/plosmedicine/article?id=10.1371/journal.pmed.1001977

[3] Molinari, N.A.M., Ortega-Sanchez, I.R., Messonnier, M.L., Thompson, W.W., Wortley, P.M., Weintraub, E. and Bridges, C.B. (2007) The Annual Impact of Seasonal Influenza in the US: Measuring Disease Burden and Costs. Vaccine, 25, 5086-5096. https://doi.org/10.1016/j.vaccine.2007.03.046

[4] Nair, H., Brooks, W.A., Katz, M., Roca, A., Berkley, J.A., Madhi, S.A. and Campbell, H. (2011) Global Burden of Respiratory Infections Due to Seasonal Influenza in Young Children: A Systematic Review and Meta-Analysis. The Lancet, 378, 1917-1930. https://doi.org/10.1016/S0140-6736(11)61051-9

[5] Uhart, M., Bricout, H., Clay, E. and Largeron, N. (2016) Public Health and Economic Impact of Seasonal Influenza Vaccination with Quadrivalent Influenza Vaccines Compared to Trivalent Influenza Vaccines in Europe. Human Vaccines \& Immunotherapeutics, 12, 2259-2268. https://doi.org/10.1080/21645515.2016.1180490

[6] Newall, A.T. and Scuffham, P.A. (2008) Influenza-Related Disease: The Cost to the Australian Healthcare System. Vaccine, 26, 6818-6823. https://doi.org/10.1016/j.vaccine.2008.09.086

[7] Cheng, P.Y., Palekar, R., Azziz-Baumgartner, E., Iuliano, D., Alencar, A.P., Bresee, J. and Widdowson, M.A. (2015) Burden of Influenza-Associated Deaths in the Americas, 2002-2008. Influenza and Other Respiratory Viruses, 9, 13-21. https://doi.org/10.1111/irv.12317

[8] Ng, S. and Gordon, A. (2015) Influenza Burden and Transmission in the Tropics. Current Epidemiology Reports, 2, 89-100. https://doi.org/10.1007/s40471-015-0038-4

[9] Simmerman, J.M. and Uyeki, T.M. (2008) The Burden of Influenza in East and South-East Asia: A Review of the English Language Literature. Influenza and Other Respiratory Viruses, 2, 81-92. https://doi.org/10.1111/j.1750-2659.2008.00045.x

[10] Yang, L., Ma, S., Chen, P.Y., He, J.F., Chan, K.P., Chow, A. and Peiris, J.M. (2011) 
Influenza Associated Mortality in the Subtropics and Tropics: Results from Three Asian Cities. Vaccine, 29, 8909-8914. https://doi.org/10.1016/j.vaccine.2011.09.071

[11] Azziz Baumgartner, E., Dao, C.N., Nasreen, S., Bhuiyan, M.U., Mah-E-Muneer, S., Mamun, A.A. and Bresee, J. (2012) Seasonality, Timing, and Climate Drivers of Influenza Activity Worldwide. The Journal of Infectious Diseases, 206, 838-846. https://doi.org/10.1093/infdis/jis467

[12] Saha, S., Chadha, M., Al Mamun, A., Rahman, M., Sturm-Ramirez, K., Chittaganpitch, M. and Lal, R.B. (2014) Influenza Seasonality and Vaccination Timing in Tropical and Subtropical Areas of Southern and South-Eastern Asia. Bulletin of the World Health Organization, 92, 318-330. https://doi.org/10.2471/BLT.13.124412

[13] Durand, L.O., Cheng, P.Y., Palekar, R., Clara, W., Jara, J., Cerpa, M. and Azziz-Baumgartner, E. (2016) Timing of Influenza Epidemics and Vaccines in the American Tropics, 2002-2008, 2011-2014. Influenza and Other Respiratory Viruses, 10, 170-175. https://doi.org/10.1111/irv.12371

[14] Hirve, S., Newman, L.P., Paget, J., Azziz-Baumgartner, E., Fitzner, J., Bhat, N. and Zhang, W. (2016) Influenza Seasonality in the Tropics and Subtropics-When to Vaccinate? PLoS ONE, 11, e0153003. https://pubmed.ncbi.nlm.nih.gov/27119988/ https://doi.org/10.1371/journal.pone.0153003

[15] Guo, D., Li, K.C., Peters, T.R., Snively, B.M., Poehling, K.A. and Zhou, X. (2015) Multi-Scale Modeling for the Transmission of Influenza and the Evaluation of Interventions toward it. Scientific Reports, 5, Article No. 8980. https://doi.org/10.1038/srep08980

[16] Won, M., Marques-Pita, M., Louro, C. and Gonçalves-Sá, J. (2017) Early and Real-Time Detection of Seasonal Influenza Onset. PLoS Computational Biology, 13, e1005330. https://doi.org/10.1371/journal.pcbi.1005330

[17] Snacken, R., Broberg, E., Beauté, J., Lozano, J.E., Zucs, P. and Amato-Gauci, A.J. (2014) Influenza Season 2012-2013 in Europe: Moderate Intensity, Mixed (Sub) Types. Epidemiology \& Infection, 142, 1809-1812. https://doi.org/10.1017/s0950268814001228

[18] Hale, B.G., Albrecht, R.A. and García-Sastre, A. (2010) Innate Immune Evasion Strategies of Influenza Viruses. Future Microbiology, 5, 23-41. https://doi.org/10.2217/fmb.09.108

[19] Lofgren, E., Fefferman, N.H., Naumov, Y.N., Gorski, J. and Naumova, E.N. (2007) Influenza Seasonality: Underlying Causes and Modeling Theories. Journal of Virology, 81, 5429-5436. https://doi.org/10.1128/JVI.01680-06

[20] Tay, E.L., Grant, K., Kirk, M., Mounts, A. and Kelly, H. (2013) Exploring a Proposed WHO Method to Determine Thresholds for Seasonal Influenza Surveillance. PLoS $O N E$, 8, e77244. https://doi.org/10.1371/journal.pone.0077244

[21] Steiner, S.H., Grant, K., Coory, M. and Kelly, H.A. (2010) Detecting the Start of an Influenza Outbreak Using Exponentially Weighted Moving Average Charts. BMC Medical Informatics and Decision Making, 10, Article No. 37. https://doi.org/10.1186/1472-6947-10-37

[22] World Health Organization (2017) Pandemic Influenza Severity Assessment (PISA): A WHO Guide to Assess the Severity of Influenza in Seasonal Epidemics and Pandemics.

https://scholar.google.fr/scholar?cluster $=17630463871277389421 \&$ hl $=$ fr\&as $\quad$ sdt $=20$ $\underline{05 \& \text { sciodt }=0,5}$

[23] World Health Organization (2011) Strengthening Response to Pandemics and Other Public-Health Emergencies: Report of the Review Committee on the Functioning of 
the International Health Regulations (2005) and on Pandemic Influenza (H1N1) 2009.

https://scholar.google.com/scholar? cluster $=2990743697744802456 \& \mathrm{hl}=\mathrm{fr} \&$ as $\mathrm{sdt}=0$ 25

[24] World Health Organization (2013) Global Epidemiological Surveillance Standards for Influenza.

https://scholar.google.com/scholar? cluster $=17107875054084040652 \& \mathrm{hl}=$ fr\&as $\mathrm{sdt}=$ $\underline{0,5}$

[25] Murray, J.L., Marques, D.F., Cameron, R.L., Potts, A., Bishop, J., von Wissmann, B. and McMenamin, J. (2018) Moving Epidemic Method (MEM) Applied to Virology Data as a Novel Real Time Tool to Predict Peak in Seasonal Influenza Healthcare Utilisation. The Scottish Experience of the 2017/18 Season to Date. Eurosurveillance, 23, Article ID: 18-00079. https://doi.org/10.2807/1560-7917.ES.2018.23.11.18-00079

[26] Rakocevic, B., Grgurevic, A., Trajkovic, G., Mugosa, B., Grujicic, S.S., Medenica, S. and Vega, T. (2019) Influenza Surveillance: Determining the Epidemic Threshold for Influenza by Using the Moving Epidemic Method (MEM), Montenegro, 2010/11 to 2017/18 Influenza Seasons. Eurosurveillance, 24, Article ID: 1800042. https://doi.org/10.2807/1560-7917.ES.2019.24.12.1800042

[27] Vega, T., Lozano, J.E., Meerhoff, T., Snacken, R., Mott, J., Ortiz de Lejarazu, R. and Nunes, B. (2013) Influenza Surveillance in Europe: Establishing Epidemic Thresholds by the Moving Epidemic Method. Influenza and Other Respiratory Viruses, 7, 546-558. https://doi.org/10.1111/j.1750-2659.2012.00422.x

[28] Abd El Gawad, B., Vega, T., El Houssinie, M., Mohsen, A., Fahim, M., El Sood, H.A. and Refaey, S. (2020) Evaluating Tools to Define Influenza Baseline and Threshold Values Using Surveillance Data, Egypt, Season 2016/17. Journal of Infection and Public Health, 13, 430-437. https://doi.org/10.1016/j.jiph.2019.05.005

[29] Fleming, D.M., Zambon, M., Bartelds, A.I.M. and De Jong, J.C. (1999) The Duration and Magnitude of Influenza Epidemics: A Study of Surveillance Data from Sentinel General Practices in England, Wales and the Netherlands. European Journal of Epidemiology, 15, 467-473. https://doi.org/10.1023/A:1007525402861

[30] Vega, T., Lozano, J.E., Meerhoff, T., Snacken, R., Beauté, J., Jorgensen, P. and Brown, C. (2015) Influenza Surveillance in Europe: Comparing Intensity Levels Calculated Using the Moving Epidemic Method. Influenza and Other Respiratory Viruses, 9, 234-246. https://doi.org/10.1111/irv.12330

[31] Centers for Disease Control and Prevention (2017) Overview of Influenza Surveillance in the United States. https://www.cdc.gov/flu/weekly/overview.htm

[32] Ly, S., Arashiro, T., Ieng, V., Tsuyuoka, R., Parry, A., Horwood, P. and Arima, Y. (2017) Establishing Seasonal and Alert Influenza Thresholds in Cambodia Using the WHO Method: Implications for Effective Utilization of Influenza Surveillance in the Tropics and Subtropics. Western Pacific Surveillance and Response Journal, 8, 22-32.

[33] Muyembe Tamfum, J.J., Nkwembe, E., Bi Shamamba, S.K., Bankoshi, F., Ilunga, B.K., Katz, K.A. and Wemankoy, E.O. (2012) Sentinel Surveillance for InfluenzaLike Illness, Severe Acute Respiratory Illness, and Laboratory-Confirmed Influenza in Kinshasa, Democratic Republic of Congo, 2009-2011. The Journal of Infectious Diseases, 206, 36-40. https://doi.org/10.1093/infdis/jis537

[34] Bangert, M., Gil, H., Oliva, J., Delgado, C., Vega, T., De Mateo, S. and Larrauri, A. (2017) Pilot Study to Harmonize the Reported Influenza Intensity Levels within the Spanish Influenza Sentinel Surveillance System (SISSS) Using the Moving Epidemic 
Method (MEM). Epidemiology \& Infection, 145, 715-722.

https://doi.org/10.1017/S0950268816002727

[35] Cooper, D.L., Verlander, N.Q., Elliot, A.J., Joseph, C.A. and Smith, G.E. (2009) Can Syndromic Thresholds Provide Early Warning of National Influenza Outbreaks? Journal of Public Health, 31, 17-25. https://doi.org/10.1093/pubmed/fdm068 